\title{
Study of stress-induced velocity variation in concrete under direct tensile force and monitoring of the damage level by using thermally-compensated Coda Wave Interferometry
}

\author{
Yuxiang Zhang a , Odile Abraham ${ }^{\mathrm{a}}$, Frédéric Grondin ${ }^{\mathrm{b}}$, Ahmed Loukili ${ }^{\mathrm{b}}$, Vincent Tournat ${ }^{\mathrm{c}}$, Alain Le Duff ${ }^{\mathrm{d}}$, \\ Bertrand Lascoup ${ }^{\mathrm{e}}$, Olivier Durand ${ }^{\mathrm{a}}$ \\ a LUNAM Université, IFSTTAR, MACS, CS4, F-44344 Bouguenais Cedex, France \\ ${ }^{\mathrm{b}} \mathrm{GeM}, \mathrm{CNRS}$ 6183, Ecole Centrale de Nantes, 1 rue de la Noë, 44321 Nantes, France \\ ${ }^{\mathrm{c}}$ LAUM, CNRS 6613, Université du Maine, Avenue O. Messiaen, 72085 Le Mans, France \\ ${ }^{\mathrm{d}}$ Groupe ESEO, 4 rue Merlet de la Boulaye, 49009 Angers, France \\ e ESTACA, Parc Universitaire Laval-Changé, Rue Georges Charpak, 53061 Laval, France
}

In this paper, we describe an experimental study of concrete behavior under a uniaxial tensile load by use of the thermally-compensated Coda Wave Interferometry (CWI) analysis. Under laboratory conditions, uniaxial tensile load cycles are imposed on a cylindrical concrete specimen, with continuous ultrasonic measurements being recorded within the scope of bias control protocols. A thermally-compensated CWI analysis of multiple scattering waves is performed in order to evaluate the stress-induced velocity variation. Concrete behavior under a tensile load can then be studied, along with CWI results from both its elastic performance (acoustoelasticity) and plastic performance (microcracking corresponding to the Kaiser effect). This work program includes a creep test with a sustained, high tensile load; the acoustoelastic coefficients are estimated before and after conducting the creep test and then used to demonstrate the effect of creep load.

\author{
Keywords: \\ Coda Wave Interferometry \\ Creep damage \\ Concrete \\ Acoustoelasticity \\ Microcracking
}

\section{Introduction}

Concrete is the most widely used man-made construction material in the world [1]. It has been extensively used for several decades in the field of civil engineering. For safety reasons and to ensure optimal asset management, the demand for monitoring concrete performance and inspecting concrete deterioration has been strong. In order to meet such demand, considerable progress has been achieved in the development of Non-Destructive Testing (NDT) methods [2,3]. As one of these NDT methods and because it uses the ultrasonic multiple scattered waves caused by high concrete heterogeneity, Coda Wave Interferometry (CWI) shows great sensitivity to perturbations in concrete structures [4,5], which makes this method suitable for structural evaluation and monitoring.

As a viscoelastic material, concrete exhibits a nonlinear elasticity that can be observed from the stress-dependent propagation velocity of elastic waves propagating within the material [6]. Experiments under compressive load [7,8] demonstrate that acoustoelastic theory may provide an explanation of the stress-induced velocity variation in the elastic wave propagation of concrete. Moreover, previous studies $[9,10]$ have also shown that the nature of concrete, i.e. as a quasi-fragile material, makes it easily damaged by the applied stress, which could also cause changes in propagation velocity. This stress-induced damage is a common reason behind concrete deterioration, and the detection of stress-induced damage is a major problem encountered in the non-destructive testing of concrete.

The objective of this paper is to experimentally study the behavior of concrete under direct tensile load, with two sequential objectives: (1) first, separating the acoustoelastic effect and microcracking from all stress-induced velocity variations in concrete, and then estimating the effective acoustoelastic coefficient and (2) based on these results, detecting and analyzing the damage caused by a sustained high-level tensile load (creep in tension). After introducing the theoretical background in the following section, the measurement system, experimental set-up and loading procedures will be described in Section 3. All of Section 4 will be devoted to an analysis of the CWI experimental results.

\section{Theoretical background}

\subsection{Coda Wave Interferometry}

The Coda Wave Interferometry (CWI) technique introduces the heterogeneous propagation medium, e.g. concrete, as an 
interferometer in order to detect the time-scale perturbation [11]. Due to the multiple scattering effect caused by heterogeneities, the propagation paths of acoustic coda waves (i.e. multiple scattered waves) are long and complex. The use of multiple scattered waves makes the CWI analysis highly sensitive to a small perturbation of the propagation medium [12,13]. The high heterogeneity of concrete favors the generation of acoustic coda waves. An experimental study [7] has shown that a CWI analysis carried out on concrete can provide a precise evaluation of the propagation velocity variation $(0.001 \%)$. This result confirms the potential of CWI analysis as a promising non-destructive method.

The signal processing approach of CWI analysis, performed as part of this study, is called Stretching [14]; this approach relies on the idea of stretching/compressing the time axis of an ultrasonic record to simulate a constant decrease/increase of propagation velocity. Two coda signals $h_{0}$ and $h_{1}$, recorded respectively before and after a propagation velocity perturbation, are required to evaluate the velocity variation. By compressing/stretching the coda record $h_{0}$ from $h_{0}[t]$ to $h_{0}[t(1+\tau)]$, a velocity variation from $v_{0}$ to $v_{0}(1+\tau)$ can be simulated. This stretched signal $h_{0}[t(1+\tau)]$ is then compared to $h_{1}[t]$, which has been recorded with a perturbed propagation velocity $v_{1}$; next, their similarity is evaluated using the normalized cross-correlation $C C(\tau)$ :

$C C_{\left(h_{0}, h_{1}\right)}^{\left(t_{1}, t_{2}\right)}(\tau)=\frac{\int_{t_{1}}^{t_{2}} h_{0}(t(1+\tau)) \cdot h_{1}(t) d t}{\sqrt{\int_{t_{1}}^{t_{2}} h_{0}^{2}(t(1+\tau)) d t \int_{t_{1}}^{t_{2}} h_{1}^{2}(t) d t}}$

$\left[t_{1}, t_{2}\right]$ indicates the studied time window, which for the present research has been set at [300 $\mu \mathrm{s}, 500 \mu \mathrm{s}]$. The value of $\tau$ that maximizes $C C(\tau)$ is denoted $\alpha$, in indicating the velocity variation between two records in relative terms as: $\alpha=\left(v_{1}-v_{0}\right) / v_{0}$.

Due to the scattering effect, acoustic energy is converted between different types of waves during the propagation. As a superposition of all multiple scattered waves, coda signals contain energy contributions from all types of waves. Consequently, the value of $\alpha$ extracted via CWI, represents the relative variation of an average propagation velocity of the coda signal. This variation results from all the changes in propagation velocities of the different modes contributing to the coda signal.

\subsection{Acoustoelastic theory}

Given an infinitesimal deformation, the classical linear theory of elasticity is limited to a perfectly elastic material, in which the elastic modulus remains constant with the static stress level. When the material is nonlinearly elastic, the stress-strain relationship derived from the classical nonlinear elasticity becomes nonlinear, with quadratic terms in strain. In this case, instead of being constant, the elastic modulus varies with the static stress level, as well as the wave propagation velocity. To describe this nonlinear elasticity, the third-order elastic constants are introduced into the expression of elastic modulus, which extends the expression of stress-strain relationships from a linear approximation to a quadratic one [15]. One version of these third-order elastic constants has been developed by Murnaghan and are denoted $(m, n, l)[16]$.

In combination with the motion equation, Murnaghan's constants were used by Hughes and Kelly [17] to express the stressdependent propagation velocity in an acoustoelastic effect for Lagrangian coordinates. The propagation medium is here assumed to be isotropic, in which a triaxial stress field (whose axes are denoted 1,2 and 3 ) is considered homogeneous. In the case of a uniaxial load along direction 1 , the stress components can be described as: $\gamma_{1}=\sigma$ and $\gamma_{2}=\gamma_{3}=-v \sigma$, where $v$ is Poisson's ratio. Under the stress field $\left(\gamma_{1}, \gamma_{2}\right.$ and $\left.\gamma_{3}\right)$ created by the uniaxial load, velocity may be generalized as a function of stress level $\sigma$ as: $v_{i j}=v_{i j}^{0}\left(1+\beta_{i j} \cdot \sigma\right)$, where $\beta_{i j}$ is the acoustoelastic coefficient, $v_{i j}^{0}$ is the initial propagation velocity corresponding to the initial stress state, and $i, j$ represent respectively the wave propagation direction and the particle polarization direction [8]. As an intrinsic nonlinear parameter, $\beta_{i j}$ depends solely on the second- and third-order elastic constants [18] and indicates the level of nonlinearity in material elasticity. The stress-induced velocity variations $\alpha_{i j}(\sigma)$ described by acoustoelastic theory can be expressed as follows:

$\alpha_{i j}(\sigma)=\frac{\left(v_{i j}(\sigma)-v_{i j}^{0}\right)}{v_{i j}^{0}}=\beta_{i j} \cdot \sigma$

Since CWI analysis provides the modifications of the average propagation velocity $(\alpha)$ rather than velocity variation of each specific type of waves $\left(\alpha_{i j}\right)$, acoustoelastic coefficients $\beta_{i j}$ cannot be acquired individually. As an alternative, an effective value of acoustoelastic coefficient $\beta$ can be estimated from CWI's result $(\alpha)$ as $\beta=\frac{\alpha^{F}}{F}$, where the stress level is associated to the load magnitude $F$. This effective acoustoelastic coefficient can be considered as a weighted average value of all acoustoelastic coefficients $\beta=\sum e_{i j} \cdot \beta_{i j}$, where the weight coefficients $e_{i j}$ depend on the energy distribution ratio of each type of waves. This linear relationship has been confirmed experimentally on concrete by Larose and Hall [7], and the dependency between its slope and concrete's damage level has been recently exhibited [19].

According to the results of previous studies in which concrete undergoes a uniaxial compressive stress, within its elastic regime, the propagation velocities increase for $P$-waves, $S$-waves $[6,8,20]$, surface waves [9] and multiple scattered waves $[7,10]$. For the research presented herein, instead of introducing a compressive load, the concrete specimen has been subjected to a direct uniaxial tensile force, leading to the expectation of a decrease in propagation velocity with tensile load magnitude.

\subsection{Other causes of stress-induced velocity variation}

Acoustoelastic theory explains the velocity variation due to elastic deformation of the material while undergoing an applied stress, yet this is not the only contribution of stress-induced velocity variation.

Concrete is a consolidated heterogeneous material made of water, cement and aggregates. Its behavior depends on the magnitude of the applied stress. For a low level stress (typically lower than $30 \%$ of the ultimate strength), concrete stays in its elastic regime, where concrete exhibits an elastic behavior in common mechanical (macro) tests (laser displacement sensors or extensometer gauges). However, from a microscopic point of view, microcracking exists in this regime even before any loading, but its influence on macroscopic elasticity remains unnoticed with conventional measurements. With increasing stress magnitude $>30 \%$ of the ultimate strength), concrete enters in its quasi-brittle regime, where bond cracks increase rapidly in length, width and numbers [21], and eventually become large defect that can alter elasticity of concrete. Comparing to the common mechanical measurement devices, which do not detect any damage until the maximum load is reached, some nondestructive methods are sensitive to the microcracking process and capable to measure an irreversible process $[9,10]$. In addition, an intermediate regime is reported to exist between the elastic and brittle regimes as a time-based slow dynamic effect. This effect has been studied by implementing a CWI analysis [22].

Microcracks are believed to be a cause of propagation velocity reduction, and this can be observed as of the early phase of material deterioration or structural damage $[23,24]$, which makes this observation critical to structural health monitoring. By measuring the surface wave velocity variation, Shokouhi et al. [9] revealed 
that the initiation of microcracking, caused by a uniaxial compressive load, follows the Kaiser effect, which explains the capability of concrete to "remember" the previous maximum stress level ever experienced. Once the current stress level exceeds the previous maximum value, modification of the microstructure begins, resulting in the initiation of new microcracks. The Kaiser effect on concrete has also been observed by use of the acoustic emission method [24].

\section{Experimental configuration}

The GeM Institute (Ecole Centrale de Nantes) has been studying the influence of basic creep in the residual capacity of concrete for many years $[25,26]$. To assess damage evolution in concrete under a tensile load, a new experimental device has been developed. Details on this effort are provided in. An experimental procedure calls for coupling with the CWI analysis in order to monitor damage evolution in concrete under a sustained load.

\subsection{Materials}

The concrete specimens were mixed with Portland cement CPACEMII 42.5 and crushed limestone aggregate distributed in fine sand, with a maximum size of $5 \mathrm{~mm}$ and density of $2570 \mathrm{~kg} / \mathrm{m}^{3}$, in addition to crushed gravel with a size of $5-12.5 \mathrm{~mm}$ and a density of $2620 \mathrm{~kg} / \mathrm{m}^{3}$. A superplasticizer agent (Glenium 21) has been added for workability. Table 1 lists the mix quantities of all constituent materials. This mixture is characterized by a water-to-cement $(\mathrm{W} / \mathrm{C})$ ratio of 0.56 and a slump of $70 \mathrm{~mm}$.

\subsection{The direct tensile test device}

This study involved the design and development of two frames of creep [27]. The specimens were fitted with two rings attached to the concrete using three set screws. Three LVDT displacement sensors, with a precision of $3 \mu \mathrm{m}$, were positioned between the two crowns and then placed on three generators at $120^{\circ}$. The relative displacement of the two sections was thus measured by following specimen elongation over a length equal to approximately half the specimen length (in our case $28 \mathrm{~cm}$ ) in the central area of the specimen to overcome edge effects.

The concrete loading step is accomplished by an electromechanical press. The force sensor has a capacity of $100 \mathrm{kN}$, which is sufficient for tensile testing on concrete and mortar specimens with lower tensile strength. The test can be controlled by either force or cylindrical motion, with speeds of between $10^{-6}$ and $10^{-1} \mathrm{~mm} / \mathrm{s}$. The specimen is fastened to the frame through steel helmets attached to the frame on one side and glued to the specimen on the other. The development of specimen bonding through the helmets is critical to test success. To improve the cohesion between headset and specimen, the specimens were subjected to a slight compressive force $(0.2 \mathrm{kN})$ through the cylinder before applying the glue. Bonding took place $24 \mathrm{~h}$ before the tensile test, at which time the glue was fully dry.

Table 1

Concrete mix composition.

\begin{tabular}{ll}
\hline Constituents & Dosage $\left(\mathrm{kg} / \mathrm{m}^{3}\right)$ \\
\hline Gravel: G5/12.5 mm & 936.0 \\
Sand: 0/5 mm & 780.0 \\
Cement: CEMII 42.5 & 350.0 \\
Water & 219.5 \\
Superplasticizer & 1.9 \\
\hline
\end{tabular}

\subsection{Thermally-compensated CWI measurement system}

Similarly to other ultrasonic techniques, the CWI analysis of velocity evolution is subjected to thermal bias due to ambient temperature fluctuations [28-30]. An alternative bias control technique for CWI analysis has been applied in this research in order to reduce bias originating from temperature, experimental conditions and signal processing. This technique involves the use of two identical concrete specimens: the first one called "test specimen" is subjected to tensile load, and the second one, called the "reference specimen", stays load-free and is placed nearby the test specimen to experience the same temperature fluctuations. The coda measurements are recorded simultaneously on both specimens sharing the same acquisition system (Fig. 1).

Two ultrasound transducers (containing source and receiver) were glued on opposite sides in the middle of each specimen. A 200- $\mu$ s chirp signal with central frequency of $500 \mathrm{kHz}$ was amplified to $200 \mathrm{~V}$ (peak-to-peak) and then sent into the concrete specimens. The wavelengths around this frequency were comparable to the aggregate sizes, thus ensuring the generation of multiple scattered waves. The received signal was recorded by a 16-bit data acquisition system with sampling frequency of $5 \mathrm{MHz}$; the acquisitions were synchronized with the excitation signals by $10-\mathrm{MHz}$ reference clock signals. To improve the signal-to-noise ratio, each record was the average of 16 consecutive acquisitions with a 30 $\mathrm{Hz}$ repeat frequency. Since each record required less than $1 \mathrm{~s}$, the propagation velocity can be considered constant during the recording procedure.

The recorded coda signals from both specimens are analyzed by following the exact same signal processing procedures. The CWI results $\alpha_{\text {test }}$ and $\alpha_{\text {ref }}$ are then obtained as the velocity variation in both specimens. Since $\alpha_{\text {test }}$ contains contribution from both bias and stress-induced velocity variation, and $\alpha_{\text {ref }}$ originates from bias (thermal and experimental) only, the difference $\alpha_{\text {test }}-\alpha_{\text {ref }}$ corresponds to the pure stress-induced velocity variation $\alpha_{F}$. As demonstrated in the experimental results [30], this easy-implemented technique can effectively compensates for the thermally-induced velocity variation and reduces experimental bias, thereby improving both the accuracy and reliability of CWI results. The notation $\alpha_{F}$ will be used in this paper to denote the velocity variation caused by the applied tensile load only (after bias control).

\subsection{Experimental procedure}

Cylindrical specimens $\left(11 \times 45 \mathrm{~cm}^{3}\right)$ were cast in molds and then vibrated into three layers. The specimens were maintained in a humid room at $20^{\circ} \mathrm{C}$ and $100 \%$ relative humidity. At the age of two months, they were removed from the molds and corrected in order to produce two very smooth and parallel surfaces. The specimens were then wrapped in a double layer of self-adhesive aluminum paper to prevent drying. The specimen to be submitted to creep is then placed into the two steel helmets attached to the frames. All tests were performed in a climate-controlled room at a temperature of $20^{\circ} \mathrm{C}\left( \pm 2{ }^{\circ} \mathrm{C}\right)$ and relative humidity of $50 \%( \pm 5 \%)$.

The entire experimental procedure comprised two sets of loading tests and a creep test of tensile load (Fig. 2a). The two sets of loading tests were identical and followed the same protocol, with each set including three loading tests (Fig. 2b); subscripts $s$ and $t$ represent respectively the set number and loading test number. The first two loading tests (i.e. $t=1,2$ ) in each set contained a loading phase $\left[A_{s t} \rightarrow B_{s t}\right]$, an unloading phase $\left[B_{s t} \rightarrow C_{s t}\right]$ and a 1 -h relaxation $\left[C_{s t} \rightarrow A_{s(t+1)}\right]$ so as to suppress any residual influence from the loading test (slow dynamic effect), whereas the third loading test $(t=3)$ contained only a loading phase.

The three loading tests in each set had three increasing target loads, respectively $8 \mathrm{kN}, 12 \mathrm{kN}$ and $18 \mathrm{kN}$. With an ultimate tensile 


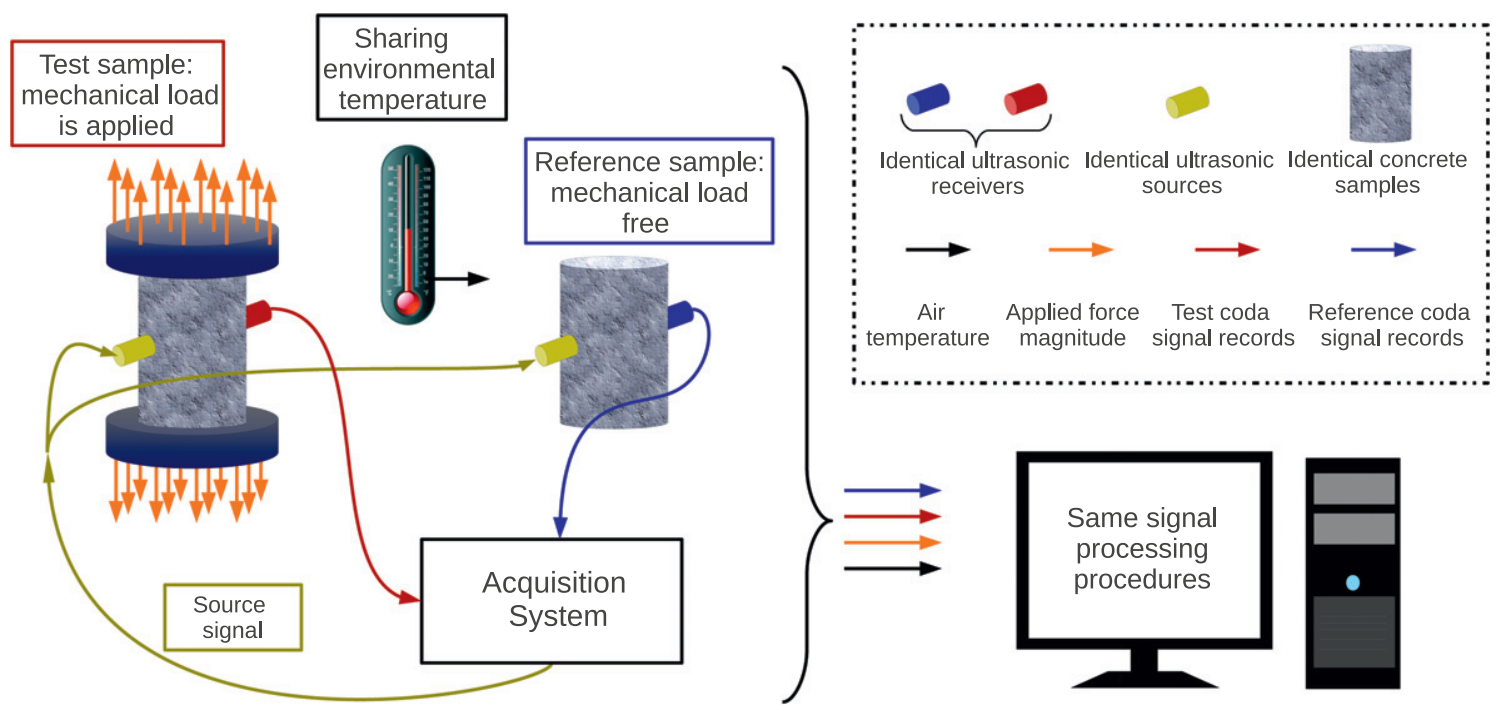

Fig. 1. Schematic representation of the CWI measurement system with bias control technique.

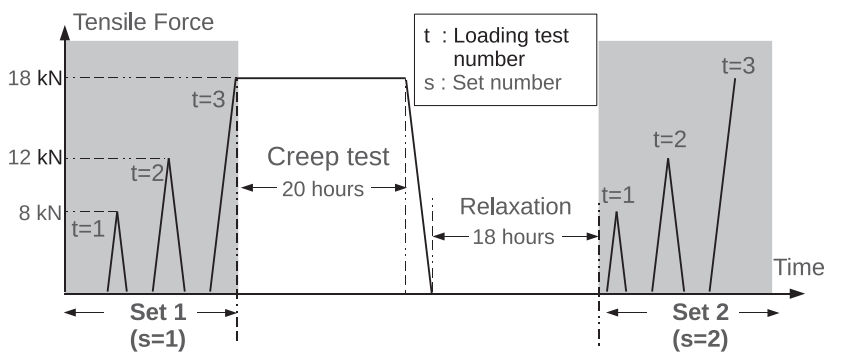

(a)

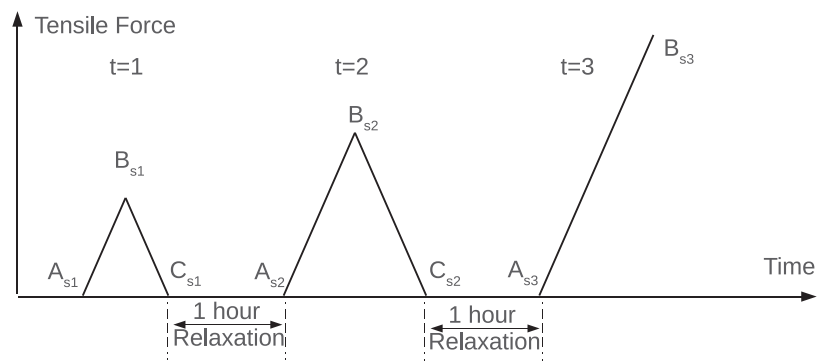

(b)

Fig. 2. Description and notations for the tensile load control: (a) designed loading procedure for the entire experiment, which comprises two sets of loading tests and one creep test and (b) detailed load control for a single set of loading tests, where $A$, $B$ and $C$ designate the key points of each test ( $A$ is the start of the loading phase, $B$ is the end of the loading phase/start of the unloading phase, and $C$ is the end of the unloading phase).

strength pre-measured at $22 \pm 1 \mathrm{kN}$, these three targets were associated with respectively $36 \%, 55 \%$ and $82 \%$ of the ultimate tensile strength of the concrete. All loading and unloading steps were performed at a speed of $32 \mathrm{kN} / \mathrm{min}$.

After the loading procedure $\left[A_{13} \rightarrow B_{13}\right]$ (Fig. 2), the applied tensile force was held at a constant $18 \mathrm{kN}$ for $20 \mathrm{~h}$ as a creep test in tension. The specimen was then left stress-free for another $18 \mathrm{~h}$, which served as a long relaxation period. This creep test was designed to generate microcracks, and the experimental results from acoustic emission testing has confirmed the damage created by this type of creep test [31].

\section{Experimental results}

\subsection{General presentation of CWI results}

The bias-controlled CWI result, $\alpha_{F}$, presents the stress-induced velocity variation (solely) in relative percentage (\%) terms as: $\alpha_{F}=\Delta v / v_{0}$, where $v_{0}$ is the propagation velocity in the initial state when the concrete is intact and stress-free, and $\Delta v$ refers to velocity changes from $v_{0}$. This reference velocity $v_{0}$ remains unchanged for all CWI results $\alpha_{F}$ obtained throughout the experiment, which makes all results of $\alpha_{F}$ or $\Delta \alpha_{F}$ proportional to an absolute velocity variation $(\mathrm{m} / \mathrm{s})$. The $\alpha_{F}$ values obtained in both sets are illustrated as a function of time in Fig. 3, along with the corresponding load levels. All $\alpha_{F}$ values at the key points $\left(A_{s t}, B_{s t}\right.$ and $C_{s t}$ in Fig. $\left.2 \mathrm{~b}\right)$ from both sets of loading tests are detailed in Table 2 .

An initial observation from Fig. 3 is that $\alpha_{F}$ follows the load level, i.e. it decreases as the tensile force is increasing. This general observation is consistent with acoustoelastic theory, though certain detailed observations cannot be fully explained by this theory alone. The applied force returns to zero after the unloading procedure and remains there throughout the relaxation period; meanwhile, the propagation velocity slowly increases without any change in applied force. This observation may be correlated with the slow dynamic effect, which has previously been studied [22] and will not be discussed any further in this paper.

A number of differences have been observed between the two sets of loading tests. In referring to both Fig. 3 and Table 2, it can be seen that a portion of the velocity reduction remains within the concrete body and accumulates after each loading test during the first set $(s=1)$, which causes a lower initial velocity at the beginning of the subsequent loading test, as $\alpha_{F}\left(A_{11}\right)>\alpha_{F}\left(A_{12}\right)>\alpha_{F}$ $\left(A_{13}\right)$. On the other hand, all initial velocities in the second set of loading tests $\alpha_{F}\left(A_{2 t}\right)(s=2 ; t=1,2$ and 3$)$ are similar. In addition, the maximum velocity decreases during the first set $(s=1)$, reaching $0.248 \%(0 \% \rightarrow-0.248 \%)$; by following the exact same protocol, the maximum velocity decrease is only $0.169 \%(-0.055 \% \rightarrow$ $-0.224 \%)$ in the second set $(s=2)$.

Acoustoelasticity cannot fully explain all stress-induced velocity variations since over the entire load range, irreversible strains take place in the concrete. These strains may be interpreted as the deterioration or damage to concrete structure, initiated through the form of distributed microscopic damage (i.e. microcracks). With 


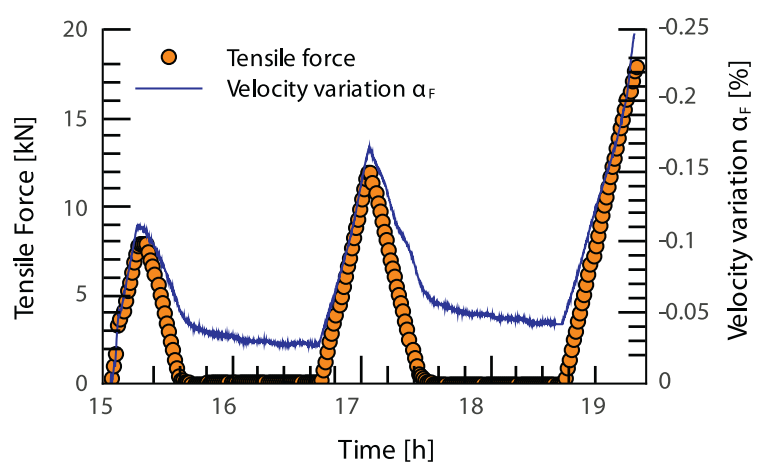

(a)

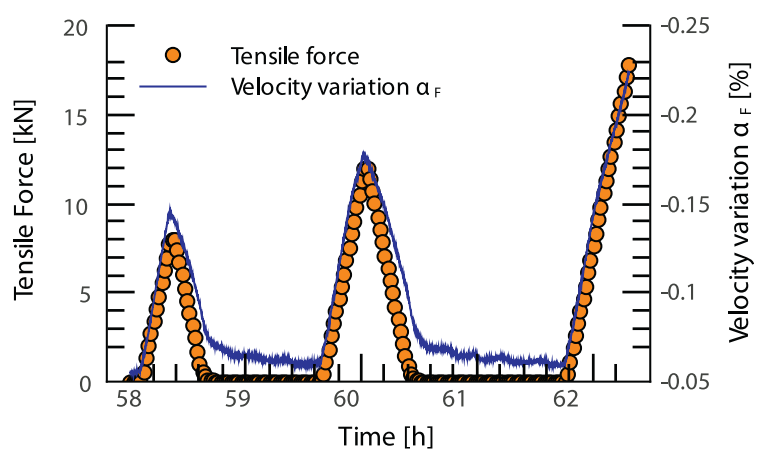

(b)

Fig. 3. Velocity variation and tensile load level vs. time during: (a) the first set of loading tests $(s=1)$ and (b) the second set of loading tests $(s=2)$; the solid circles indicate the applied tensile force, while the blue curve presents the stress-induced velocity variation $\alpha_{F}$ in relative terms.

\section{Table 2}

Velocity variation $\alpha_{F}$ at key points $(A, B$ and $C$ ) of each loading test, subscripts $s$ and $t$ are used to represent respectively the set number and loading test number (related to Fig. 2).

\begin{tabular}{ccclll}
\hline \multicolumn{1}{c}{$s$} & $t$ & Target load $(\mathrm{kN})$ & \multicolumn{3}{c}{ Velocity variation $(\%)$} \\
\cline { 4 - 6 } & & & $\alpha_{F}\left(A_{s t}\right)$ & $\alpha_{F}\left(B_{s t}\right)$ & $\alpha_{F}\left(C_{s t}\right)$ \\
\hline 1 & 1 & 8 & 0 & -0.111 & -0.052 \\
& 2 & 12 & -0.029 & -0.165 & -0.075 \\
& 3 & 18 & -0.045 & -0.248 & $\mathrm{~N} / \mathrm{A}$ \\
& 1 & 8 & -0.055 & -0.144 & -0.086 \\
& 2 & 12 & -0.060 & -0.177 & -0.086 \\
& 3 & 18 & -0.062 & -0.224 & N/A \\
\hline
\end{tabular}

an increase in load level, these microcracks might become connected and gradually develop into large defects [24,9,23].

\subsection{Kaiser effect}

The Kaiser effect is a "stress memory" effect observed in many materials; according to this effect, microcracking occurs in a material only if the load level exceeds its historical maximum ever experienced. Otherwise, no significant modification in microstructure will take place, hence no additional creation of microcracks. This effect was originally observed with an acoustic emission test conducted on rock material as well as on concrete [32]; it has also been noticed with the velocity variation of surface waves in concrete under compressive load [9]. In this paper, through the use of bias-controlled CWI analysis [30], the Kaiser effect is clearly observed via the velocity variation of multiple scattered waves of concrete under direct uniaxial tensile load.
The velocity variations obtained from the three loading phases in the first set $(s=1)$ are illustrated as a function of applied tensile force in Fig. 4a. The first observation is that the starting point (at $0 \mathrm{kN}$ ) of each curve becomes increasingly lower. The concrete is initially intact, with its historical maximum load level initially being zero. With an increasing target load ( $8 \mathrm{kN} / 12 \mathrm{kN} \nearrow 18 \mathrm{kN})$, the historical maximum has been exceeded in every loading test of the first set ( $s=1 ; t=1,2$ and 3 ); new microcracks are thus formed during every test of this first set. In considering that microcracking is irreversible and reduces propagation velocity, the creation and accumulation of microcracks may explain the decreasing initial velocity during the first set $\left(\alpha_{F}\left(A_{11}\right)>\alpha_{F}\left(A_{12}\right)>\alpha_{F}\left(A_{13}\right)\right)$. Another observation is that the curves of later loading tests intersect the previous test curves at their maximum load levels. For example, the curve of test $2(s=1 ; t=2)$ crosses the test 1 curve $(s=1 ; t=1$ with a target load of $8 \mathrm{kN}$ ) when the applied force returns to the 8-kN level. The velocity variations for these two loading phases $\left[A_{11} \rightarrow B_{11}\right]$ and $\left[A_{12} \rightarrow B_{12}\right]$ are illustrated individually in Fig. 5 in order to further explain this phenomenon.

During the first loading phase of the first set $(s=1 ; t=1)$ $\left[A_{11} \rightarrow B_{11}\right]$ (Fig. 5a), the historical maximum load $(0 \mathrm{kN})$ is exceeded from the very beginning and microcracking takes place throughout the load range. The velocity decrease is thus caused by the combined influence of the acoustoelastic effect and microcracking. At the end of this loading test $(s=1 ; t=1)$, since the applied force has returned to $0 \mathrm{kN}$ and the slow dynamic effect is considered suppressed after the 1-h relaxation, the remaining velocity reduction $\left(\alpha_{F}\left(A_{12}\right)=-0.029 \%\right)$ is believed to be caused by the creation of irreversible microcracks. By applying linear regression, a slope of $-0.01438 \% / \mathrm{kN}$ is obtained for this loading phase, which indicates the rate of velocity decrease in response to the applied tensile force.

During the second loading phase of the first set $(s=1 ; t=2)$ $\left[A_{12} \rightarrow B_{12}\right]$ (Fig. 5b), the concrete exhibits two distinct behaviors. The first behavior appears within the 0 to $8-\mathrm{kN}$ load range; given that the previous maximum load has not been exceeded, no new microcrack is formed and the velocity decrease is solely due to the acoustoelastic effect. The second behavior appears within the 8 to $12-\mathrm{kN}$ load range, over which the load level exceeds the previous maximum $(8 \mathrm{kN})$ and microcracking occurs. Similar to loading phase $\left[A_{11} \rightarrow B_{11}\right](s=1 ; t=1)$, the velocity decrease within this load range is due to the combined influence of acoustoelastic and microcracking effects. Instead of processing a single slope for entire load range, two slopes are individually calculated for the two distinct load ranges associated with the two concrete behaviors. The loading phase slope of $\Delta \alpha_{F} / \Delta F$ will be denoted hereafter $S_{s t}^{b}$, with subscript $s t$ used to identify the loading phase ( $s$ stands for the set number and the loading test number, as in Fig. 2), while superscript $b$ refers to the concrete behavior $(b=1,2)$. The two loading phases slopes $\left[A_{12} \rightarrow B_{12}\right]$ are thus equal to: $S_{12}^{1}=-0.00979 \% / \mathrm{kN}$ (behavior 1) and $S_{12}^{2}=-0.01389 \% / \mathrm{kN}$ (behavior 2).

Since the velocity variation in loading phase $\left[A_{11} \rightarrow B_{11}\right]$ $(s=1 ; t=1)$ is correlated with the second concrete behavior (see the previous section), the calculated slope can be expressed as: $S_{11}^{2}=-0.01438 \% / \mathrm{kN}$. Due to the difference in concrete behavior, the slopes obtained over the load range $[0 \mathrm{kN} \rightarrow 8 \mathrm{kN}]$ from both loading tests $(s=1 ; t=1,2)$ also differ, i.e. $S_{12}^{1}=-0.00979 \% / \mathrm{kN}$ is greater than $S_{11}^{2}=-0.01438 \% / \mathrm{kN}$, which means that in the load range from 0 to $8 \mathrm{kN}$, the velocity decrease is less in the second loading phase $(s=1 ; t=2)$ than in the first $(s=1 ; t=1)$. As shown in Fig. 4a, however, the two curves (for tests 1 and 2) intersect when the load level reaches $8 \mathrm{kN}$, meaning that at this load level $(8 \mathrm{kN})$, the propagation velocity is nearly the same in both loading tests $(s=1 ; t=1,2)$. 


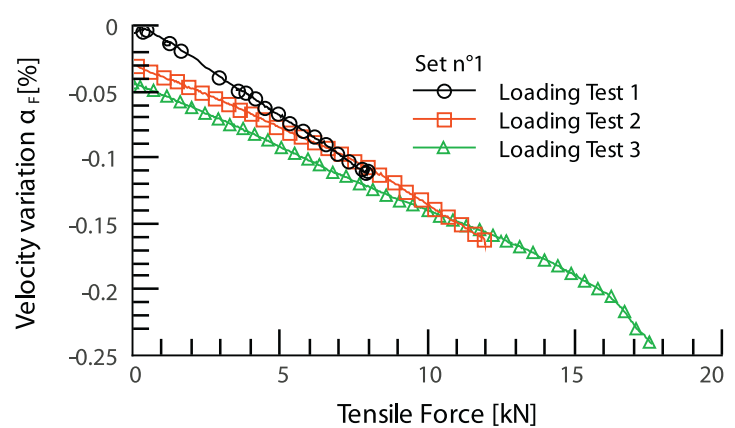

(a)

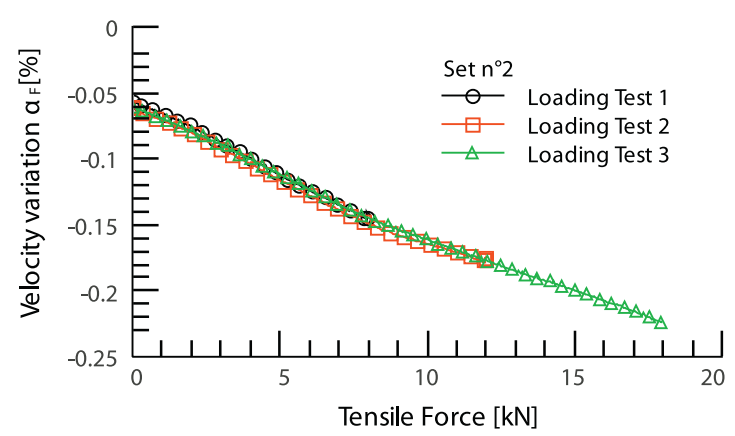

(b)

Fig. 4. Velocity variation $\alpha_{F}$ in terms of tensile force obtained during three loading phases of each set of tests.

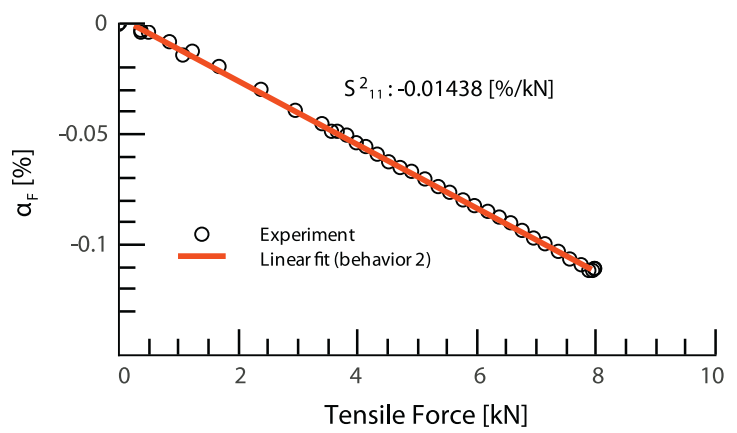

(a)

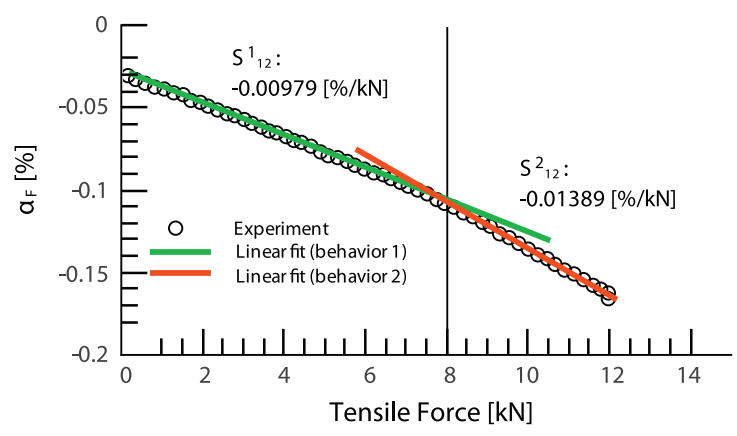

(b)

Fig. 5. Velocity variation $\alpha_{F}$ vs. tensile force for loading phases: (a) $\left[A_{11} \rightarrow B_{11}\right]$ with a target load of $8 \mathrm{kN}$ and (b) $\left[A_{12} \rightarrow B_{12}\right]$ with a target load of $12 \mathrm{kN}$; the circles are experimental CWI results of $\alpha_{F}$ and the straight lines are linear regressions. (The vertical line in image (b) is the previous maximum load level of $8 \mathrm{kN}$ and has been used to separate the two load ranges corresponding to the two concrete behaviors.)

The reason for this finding is that since microcracking occurs during the first loading test $(s=1 ; t=1)$, it causes a continuous decrease in propagation velocity throughout the loading phase. During the second loading test $(s=1 ; t=2)$ however, no velocity reduction is caused by microcracking within this range $[0 \mathrm{kN} \rightarrow 8 \mathrm{kN}$ ], which explains why the velocity decreases more slowly in this second loading test than in the first. Yet the microcracks previously created (during the first loading test) remain in the body of the concrete and cause a lower initial velocity at the beginning of the second test, as $\alpha_{F}\left(A_{12}\right)=-0.029 \%$ (Table 1 ). When the load level reaches $8 \mathrm{kN}$ in the second loading phase $(s=1 ; t=1,2)$, it can be concluded that: (1) the acoustoelastic effect is causing the same velocity variation in both loading tests and (2) since no new microcracking is occurring during the second loading test, these same microcracks are causing the same velocity reduction. Eventually, the propagation velocity will be reduced to the same level in both loading tests, and the two curves will intersect.

A similar observation can be made when examining the third loading test $(s=1 ; t=3)$, as the curve crosses the test 2 curve when the load level reaches $12 \mathrm{kN}$ (Fig. 4a). This same process has been adopted to calculate $S_{13}^{1}$ and $S_{13}^{2}$; all slopes are listed in Table 3. The slopes corresponding to the second concrete behavior (combined influence of acoustoelastic effect and microcracking) $S_{11}^{2}, S_{12}^{2}$ and $S_{13}^{2}$ indicate a very similar value, i.e. around $-0.0144 \% / \mathrm{kN}$. The slopes corresponding to the first concrete behavior (influence from the acoustoelastic effect alone), as $S_{12}^{1}$ and $S_{13}^{1}$, show values around $-0.0097 \% / \mathrm{kN}$. This result is consistent with the fact that microcracks slow propagation velocity.

The identical loading tests were then conducted in the second set of loading tests. According to the Kaiser effect, since the historical maximum load level ( $18 \mathrm{kN}$ at $B_{13}$ ) had never been exceeded, the concrete performance during the entire second set of tests $(s=2)$ can be identified as the influence of the acoustoelastic effect only (i.e. the second behavior). Since no new microcracks had formed, velocity variations are due solely to the acoustoelastic effect; therefore, all processes should be elastic and reversible. The experimental results presented in Fig. 4b display a fair level of agreement, as velocity variations in all three loading phases $\left[A_{2 t} \rightarrow B_{2 t}\right](t=1,2$ or 3$)$ follow the same path. Despite the difference in load ranges, the three curves nearly overlap (Fig. 4b). Moreover, the reproducibility of our experiment and reliability of thermally-compensated CWI analysis have been confirmed.

\subsection{Effective acoustoelastic coefficient under direct tensile load}

The classical theory of acoustoelasticity is suitable for homogeneous materials with a classical nonlinear elastic behavior. The first nonlinear terms in the stress-strain relationship are quadratic in strain, which provides a linear velocity-stress relation at the first order. In concrete, a linear velocity-stress relation has been experimentally observed under uniaxial compressive load $[6,7,9,10,20]$, when the load level is typically limited within $30 \%$ of its ultimate strength (6.6 kN in our case). We observe also, for tensile load, a linear velocity-stress relation (Fig. 3). In the applied load range, concrete's behavior is in agreement with the classical theory of acoustoelasticity.

Table 3

Linear slopes of $\Delta \alpha_{F} / \Delta F$ for two behaviors of concrete obtained in the first set of loading tests.

\begin{tabular}{lll}
\hline \multirow{2}{*}{ Loading test number $(t)$} & \multicolumn{2}{l}{ Loading phase slope $(\% / \mathrm{kN})$} \\
\cline { 2 - 3 } & $S_{1 t}^{1}$ (behavior 1) & $S_{1 t}^{2}$ (behavior 2) \\
\hline 1 & & $-0.01438 \pm 0.00003$ \\
2 & $-0.00978 \pm 0.00002$ & $-0.01389 \pm 0.00005$ \\
3 & $-0.00971 \pm 0.00002$ & $-0.01485 \pm 0.00024$ \\
\hline
\end{tabular}


However, due to the observed Kaiser effect, velocity variations are interpreted to originate not only from the acoustoelastic effect, but also from processes such as micro-cracking, which manifest in the Kaiser effect. In order to extract the velocity variations originating only from the acoustoelastic effect, data from the first behavior of concrete are used (Fig. 6a). In this case, the previous maximum applied load has not been reached again and no new micro-cracking (or other processes leading to the Kaiser effect) is activated. The extracted velocity variation provides an effective acoustoelastic coefficient.

In order to evaluate velocity changes during each loading phase, $\Delta \alpha_{F}$ (in \%) serves as the velocity variation indicator for the variation in $\alpha_{F}$ during the loading procedure from $0 \mathrm{kN}$ to $6.6 \mathrm{kN}$. In each $0.1-\mathrm{kN}$ force increment, the average values $\overline{\Delta \alpha_{s}}$ are derived from all $\Delta \alpha_{s t}$ obtained from a given set of loading tests. For example, the first value of $\overline{\Delta \alpha_{1}}$ (Fig. 6b) is the average value of $\Delta \alpha_{1 t}$ obtained from the two usable loading procedures (Fig. 6a) of the first set $(s=1 ; t=2,3)$ with tensile force $F$ increasing from 0 to $0.1 \mathrm{kN}$. The average load level $\bar{F}$ is then calculated according to the same procedure as the stress level indicator.

Ultimately, the average velocity variation $\overline{\Delta \alpha_{i}}$ is illustrated in terms of average load level $\bar{F}$ in Fig. $6 \mathrm{~b}$ and $\mathrm{c}$ as empty circles (with error bars for the standard deviation). A strong linear dependence between velocity variation and applied force level is evident in both images. The straight lines represent the fitting approximations estimated by linear regression, with the slopes of the two fitting lines being assigned as the effective acoustoelastic coefficients $\beta_{s}$, whose values are $\beta_{1}=-0.00979 \pm 0.00006 \% / \mathrm{kN}$ and $\beta_{2}=-0.01116 \pm 0.00011 \% / \mathrm{kN}$. As is readily observed, the linearity between velocity variation and tensile load level is clear for both sets, a finding that is consistent with acoustoelastic theory. The absolute values of the acoustoelastic coefficients found in the research are similar to values obtained in a similar study under compressive load $[7,10]$.

\subsection{Damage of creep in tension}

After a creep test and 18-h relaxation (Fig. 2), the propagation velocity slightly decreased, by $0.01 \%\left(\alpha\left(A_{21}\right)=-0.055 \%\right)$, compared to $\alpha\left(A_{13}\right)=-0.045 \%$ obtained before the creep test (Table 1 ). Microcracking occurred during the third loading procedure in set 1 $\left[A_{13} \rightarrow B_{13}\right]$. The microcracks formed before the creep test remained as is, causing a permanent decrease in propagation velocity; this decrease of $-0.01 \%$ is of the same order of magnitude as the velocity decrease caused by microcracking during the first two loading procedures in the first set of loading tests $(s=1 ; t=1,2)$. The conclusion can thus be drawn that the creep test has not caused any significant velocity decrease.

From another perspective, by comparing the effective acoustoelastic coefficients obtained from the two sets of loading tests $(s=1,2)$, it is found that $\beta$ has dropped by $0.00137 \% / \mathrm{kN}$, from $\beta_{1}=-0.00979 \% / \mathrm{kN}$ (before the creep test) to $\beta_{2}=-0.01116 \% / \mathrm{kN}$ (after the creep test). Since the estimated uncertainty of acoustoelastic coefficients equal one order of magnitude less than this reduction, we are of the opinion that this reduction in effective acoustoelastic coefficient offers a convincing observation of the damage created by creep testing. With the concrete being constantly subjected to a high-level tensile load (82\% of ultimate strength) for more than $20 \mathrm{~h}$, microcracks are continuously formed and possibly joined between themselves, such that the diffused microcracks may become localized macro-faults and eventually cause changes in the mechanical properties of concrete.

Two different conclusions have been derived thus far, namely: (1) the creep test causes no significant velocity decrease, which usually further implies no significant microcracking and (2) the concrete is clearly damaged by the creep test, with modifications

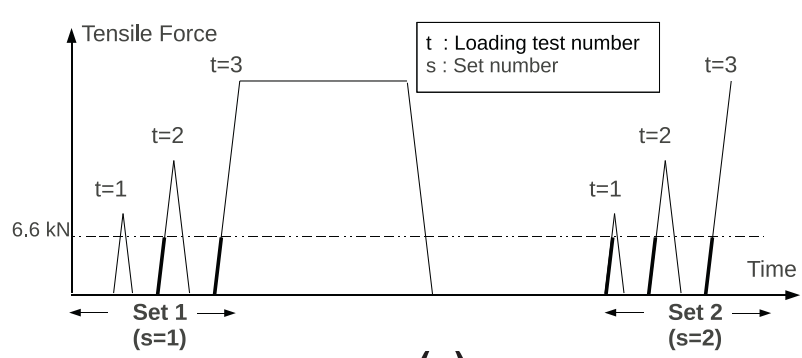

(a)

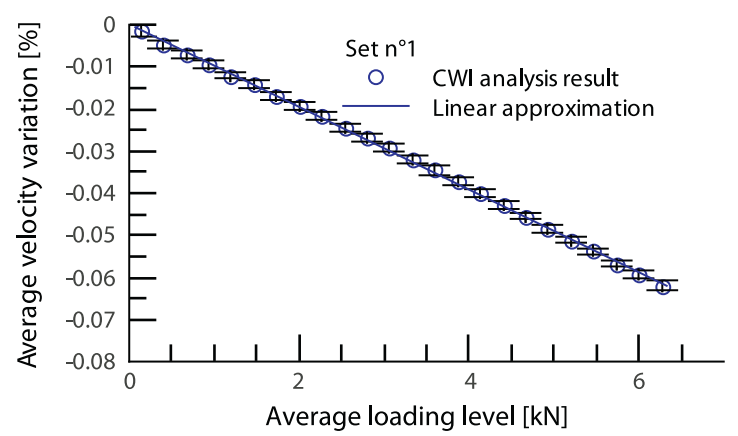

(b)

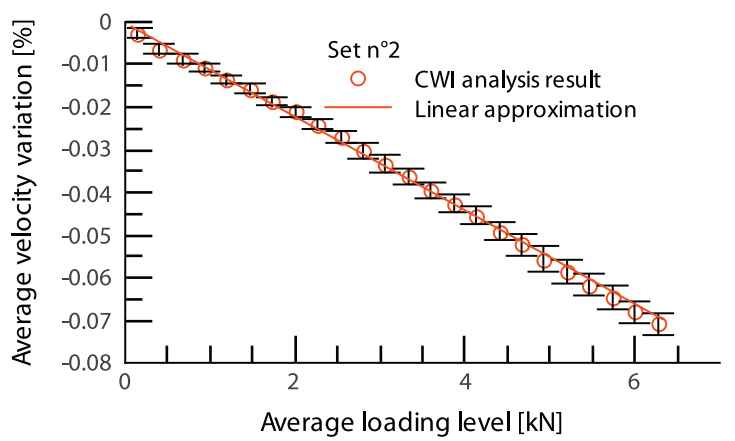

(c)

Fig. 6. Evaluation of the effective acoustoelastic coefficient: (a) presentation of loading procedures, in which CWI results are used for evaluation purposes, and (b and c) Velocity variation in terms of tensile force; the circles represent the average values of CWI results $\overline{\Delta \alpha_{F}}(\%)$, while the straight lines are the linear approximations whose slopes are assigned as the acoustoelastic coefficient.

to its mechanical properties being observed via a $14 \%$ decrease in the effective acoustoelastic coefficient. In order to understand and combine these two conclusions, the "self-healing" effect of concrete must be taken into account. Recent studies have shown a slight increase in the mechanical properties of concrete under a sustained tensile load [31]. This phenomenon is explained by the hydration of residual clinker of the cement in contact with water flowing through the formed microcracks [33]. Under these conditions therefore, a competition is playing out between this strength increase and the formation of microcracks, which tend to fragilize the material. As a result of this competition, the propagation velocity does not exhibit any significant variation. In considering that the effective acoustoelastic coefficient is a nonlinear parameter, we feel that its reduction (from $-0.00979 \% / \mathrm{kN}$ to $-0.01116 \% / \mathrm{kN}$ ) indicates that the creep test is more likely to exert an impact on the concrete material's nonlinear elastic properties.

\section{Conclusion}

In our study, we have evaluated the stress-induced velocity change in a concrete structure due to the application of a direct 
uniaxial tensile load. In being limited by the tensile strength of concrete, which is 10 times less than its compressive strength, the maximum tensile load in this study has only equaled $18 \mathrm{kN}$ (or $82 \%$ of ultimate strength). This value corresponds to an applied stress level of 1.9 MPa. Under this level of tensile stress, propagation velocity has decreased by $0.248 \%$ (due to the combined influence of acoustoelasticity and microcracking). These results provide satisfactory agreement with previous studies using a compressive load $[7,8,10]$, though with a much smaller variation scale. We must point out the high sensitivity of CWI analyses, as thanks to this sensitivity alone we can now precisely perform an evaluation of velocity changes within this small range (Fig. 3a).

Moreover, by relying on loading test cycles that follow incremental target loads, we have experimentally illustrated the Kaiser effect on concrete. By distinguishing reversible velocity variations due to elastic deformation from irreversible ones caused by microcracking, we could then illustrate the acoustoelastic effect with a linear relationship between velocity variation and tensile load level (within concrete's elastic regime), before subsequently estimating an effective acoustoelastic coefficients by means of linear regression. In this part, we must not overlook the contribution of the thermal compensation technique: without its efficient performance, a $1{ }^{\circ} \mathrm{C}$ air temperature fluctuation might be sufficient to alter the estimation result [30]. Only a test repeatability this acceptable (see Fig. 4b) can lead to a reliable and accurate estimation of the effective acoustoelastic coefficient. Due to the sustained tensile load in the creep test, this effective acoustoelastic coefficient has decreased by $14 \%$, whereas no significant reduction in propagation velocity has been observed. By taking into account the self-healing effect of concrete, we are able to deduce that the creep test is more likely to exert an impact on the nonlinear mechanical properties of concrete. By estimating an effective acoustoelastic coefficient, the monitoring of concrete's damage level can be achieved.

\section{Acknowledgements}

This research has been supported by the ECND-PdL (Loire Valley cluster for Non-Destructive Evaluation and Control), which is financed by the Loire Valley Regional government (France). Our thanks are extended to Robert Sachs, a native English speaker commissioned to proofread the final version of this paper.

\section{References}

[1] B. Lomborg, The Skeptical Environmentalist: Measuring the Real State of the World, 2001.

[2] H. Wiggenhauser, Advanced NDT methods for the assessment of concrete structures, in: H.D.F.M.P. Alexander, M.G. Beushausen (Eds.), 2nd International Conference on Concrete Repair, Rehabilitation and Retrofitting, pp. 19-30.

[3] V. Garnier, B. Piwakowski, O. Abraham, G. Villain, C. Payan, J. Chaix, Acoustical techniques for concrete evaluation: improvements, comparisons and consistencies, Constr. Build. Mater., submitted for publication.

[4] E. Larose, J. de Rosny, L. Margerin, D. Anache, P. Gouedard, M. Campillo, B. van Tiggelen, Observation of multiple scattering of khz vibrations in a concrete structure and application to monitoring weak changes, Phys. Rev. E 73 (2006) 016609.

[5] E. Larose, T. Planes, V. Rossetto, L. Margerin, Locating a small change in a multiple scattering environment, Appl. Phys. Lett. 96 (2010) 204101.

[6] T. Wu, T. Lin, The stress effect on the ultrasonic velocity variations of concrete under repeated loading, ACI Mater. J. 95 (5) (1998) 519-524.
[7] E. Larose, S. Hall, Monitoring stress related velocity variation in concrete with a $2 \times 10^{-5}$ relative resolution using diffuse ultrasound, J. Acoust. Soc. Am. 125 (2009) 1853-1856.

[8] I. Lillamand, J.-F. Chaix, M.-A. Ploix, V. Garnier, Acoustoelastic effect in concrete material under uni-axial compressive loading. NDT E Int. 43 (2010) 655-660.

[9] P. Shokouhi, A. Zoga, H. Wiggenhauser, Nondestructive investigation of stressinduced damage in concrete, Adv. Civ. Eng. 2010 (2010) 9 (Article ID 740189).

[10] S. Stahler, E. Niederleithinger, C. Sens-Schönfelder, Monitoring stress changes in a concrete bridge with coda wave interferometry, J. Acoust. Soc. Am. 129 (2011) 1945-1952.

[11] R. Snieder, A. Grt, H. Douma, J. Scales, Coda wave interferometry for estimating nonlinear behavior in seismic velocity, Science 295 (2002) 2253-2255.

[12] A. Gret, R. Snieder, J. Scales, Time-lapse monitoring of rock properties with coda wave interferometry, J. Geophys. Res. 111 (2006).

[13] V. Tournat, V.E. Gusev, Nonlinear effects for coda-type elastic waves in stressed granular media, Phys. Rev. E 80 (2009) 011306.

[14] C. Sens-Scheonfelder, E. Larose, Temporal changes in the lunar soil from correlation of diffuse vibrations, Phys. Rev. E 78 (2008) 045601.

[15] P. Rasolofosaon, B. Zinszner, P. Johnson, Propagation of elastic waves in nonlinear materials - survey of laboratory results on rock and geophysical applications, Rev. Inst. Fr. Petrol 52 (1997) 585-608.

[16] F.D. Murnaghan, Finite deformations of an elastic solid, Am. J. Math. 59 (1937) 235.

[17] D.S. Hughes, J.L. Kelly, Second-order elastic deformation of solids, Phys. Rev. 92 (1953) 1145-1149.

[18] D.M. Egle, D.E. Bray, Measurement of acoustoelastic and third-order elastic constants for rail steel, J. Acoust. Soc. Am. 60 (1976) 741-744.

[19] D.P. Schurr, J.-Y. Kim, K.G. Sabra, L.J. Jacobs, Damage detection in concrete using coda wave interferometry, NDT E Int. 44 (2011) 728-735.

[20] C. Payan, V. Garnier, J. Moysan, P.A. Johnson, Determination of third order elastic constants in a complex solid applying coda wave interferometry, Appl. Phys. Lett. 94 (2009) 011904.

[21] I. Shkolnik, Effect of nonlinear response of concrete on its elastic modulus and strength, Cem. Concr. Compos. 27 (2005) 747-757.

[22] N. Tremblay, E. Larose, V. Rossetto, Probing slow dynamics of consolidated granular multicomposite materials by diffuse acoustic wave spectroscopy, J. Acoust. Soc. Am. 127 (2010) 1239-1243.

[23] S. Selleck, E. Landis, M. Peterson, S. Shah, J. Achenbach, Ultrasonic investigation of concrete with distributed damage, ACI Mater. J. 95 (1998) 27-36.

[24] A.A. Shah, S. Hirose, Nonlinear ultrasonic investigation of concrete damaged under uniaxial compression step loading, J. Mater. Civ. Eng. 22 (2010) 476484.

[25] J. Saliba, A. Loukili, F. Grondin, J.-P. Regoin, Experimental study of creepdamage coupling in concrete by acoustic emission technique, Mater. Struct. (2012) 1-13, http://dx.doi.org/10.1617/s11527-012-9840-3.

[26] M. Omar, A. Loukili, G. Pijaudier-Cabot, Y.L. Pape, Creep-damage coupled effects: experimental investigation on bending beams with various sizes, J. Mater. Civ. Eng. 21 (2009) 65-72.

[27] J. Saliba, F. Grondin, M. Matallah, A. Loukili, H.Boussa, Relevance of a mesoscopic modelling for the coupling between creep and damage in concrete, Mech. Time-Depend. Mater., submitted for publication.

[28] A. Mazzeranghi, D. Vangi, Methodology for minimizing effects of temperature in monitoring with the acousto-ultrasonic technique, Exp. Mech. 39 (1999) 86-91, http://dx.doi.org/10.1007/BF02331110.

[29] Y. Zhang, O. Abraham, E. Larose, T. Planes, A.L. Duff, B. Lascoup, V. Tournat, R.E. Guerjouma, L.-M. Cottineau, O. Durand, Following stress level modification of real size concrete structure with coda wave interferometry (cwi), in: AIP Conference Proceedings, vol. 1335, 2011, pp. 1291-1298.

[30] Y. Zhang, O. Abraham, V. Tournat, A.L. Duff, B. Lascoup, A. Loukili, F. Grondin, O. Durand, Validation of a thermal bias control technique for coda wave interferometry (cwi), Ultrasonics, (2012), http://dx.doi.org/10.1016/ j.ultras.2012.08.003.

[31] P. Rossi, J.-L. Tailhan, F.L. Maou, L. Gaillet, E. Martin, Basic creep behavior of concretes investigation of the physical mechanisms by using acoustic emission, Cem. Concr. Res. 42 (2012) 61-73.

[32] A. Shah, Y. Ribakov, Effectiveness of nonlinear ultrasonic and acoustic emission evaluation of concrete with distributed damages, Mater. Des. 31 (2010) 37773784.

[33] S. Granger, A. Loukili, G. Pijaudier-Cabot, G. Chanvillard, Experimental characterization of the self-healing of cracks in an ultra high performance cementitious material: Mechanical tests and acoustic emission analysis, Cem. Concr. Res. 37 (2007) 519-527. 